\title{
Localized Aortic Root Dissection Secondary to Contrast Agent Injection: Feasibility of Conservative Follow-up With Echocardiography
}

\author{
Ahmet Karabulut ${ }^{1 *}$ \\ 'Department of Cardiology, Acibadem University School of Medicine, Acibadem Atakent Hospital, Istanbul, Turkey \\ *Corresponding Author: Ahmet Karabulut, MD, Associate Professor, Department of Cardiology, Acibadem University \\ School of Medicine, Acibadem Atakent Hospital, Istanbul, Turkey. Phone: +90-5053577477, Fax: +90-2124044445, \\ Email: drkarabulut@yahoo.com
}

Received December 24, 2016; Accepted January 24, 2017; Online Published February 19, 2017

\begin{abstract}
Introduction: latrogenic aortocoronary dissection (ACD) is a nightmare in interventional cardiology. Although ACD is rarely reported, the real-world prevalence is suspected of being higher due to unreported cases. The right coronary artery (RCA) ostium is involved in the majority of cases, and dissections are usually limited to the aortic sinus in half of the clinical presentations. There are different treatment strategies, including interventional approaches, surgery, and medical follow-up. Immediate stent deployment to the coronary osteum might be a life-saving procedure, and the surgical approach should be preferred in occasions of dissection extension beyond the sino-tubular junctions. Catheter trauma and subintimal progression of guidewires are major causes of ACD occurrence.

Case Presentation: Herein presented is a case report of spontaneous ACD observed after contrast injection to the RCA ostium. Approximately $8 \mathrm{cc}$ of radiocontrast agent was injected into the right coronary ostium. Antegrade and retrograde dissections which extended to the distal RCA and aortic root were observed. The right sinus of Valsalva was stained with contrast agent, and the border of the stained area was extended to the sinotubular junction. Medical follow-up was proposed by the heart team. Close follow-up with echocardiographic examination indicated the complete regression of the dissection. Conclusion: The authors consider conservative follow-up with echocardiogaphy rather than computed tomography and/or magnetic resonance to be the most appropriate imaging technique for use with stable patients.

Keywords: Aortic dissection, Radiocontrast agent, Echocardiograhy, latrogenic disease, Diagnosis
\end{abstract}

\section{Introduction}

Iatrogenic aortocoronary dissections (ACDs) are uncommon clinical entities. The majority of cases are catheter-induced. Spontaneous ACD secondary to contrast agent injection is rarely reported ${ }^{1,2}$; however, the real-world prevalance is expected to be higher because of unreported cases. Parallel to increments in complex percutaneous coronary interventions, especially retrograde chronic total occlusion procedures, the incidence of ACD is expected to become more common. Catheter trauma and subintimal progression of guidewires are major causes of ACD occurrence; isolated dissection secondary to radiocontrast injection is an extremely rare clinical entity. ${ }^{2,3}$ The right coronary artery (RCA) ostium is involved in the majority of cases, and dissections are usually limited to the aortic sinus in half of clinical presentations. There are different treatment strategies, including interventional approaches, surgery, and medical follow-up. ${ }^{4-6}$ Since early in the era of cardiovascular procedures, aortic dissections have conventionally been treated with surgery. Immediate stent deployment to the coronary osteum might be a lifesaving procedure which could prevent the progression and proximal extension of the dissection. The surgical approach should be preferred in occasions of dissection extension beyond the sino-tubular junctions. A case report of spontaneous ACD observed after contrast injection to the RCA ostium is reported herein. Treatment alternatives are discussed briefly, and the feasibility of echocardiographic follow-up is revealed.

\section{Case Presentation}

A 67-year-old male patient presented with stable angina pectoris. Coronary bypass operation had been performed on a patient eight years ago. A coronary angiography was performed through the right femoral artery, and the RCA ostium was intubated with a Judkins right catheter. Approximately $8 \mathrm{cc}$ of contrast agent (Iopamiro) was injected for right coronary imaging. Antegrade and retrograde dissections which extended to the distal RCA and aortic root were observed (Figure 1). The right sinus of

Copyright (C) 2017 The Hospital Practices and Research. This is an open-access article distributed under the terms of the Creative Commons Attribution License (http://creativecommons.org/licenses/by/4.0), which permits unrestricted use, distribution, and reproduction in any medium, provided the original work is properly cited. 


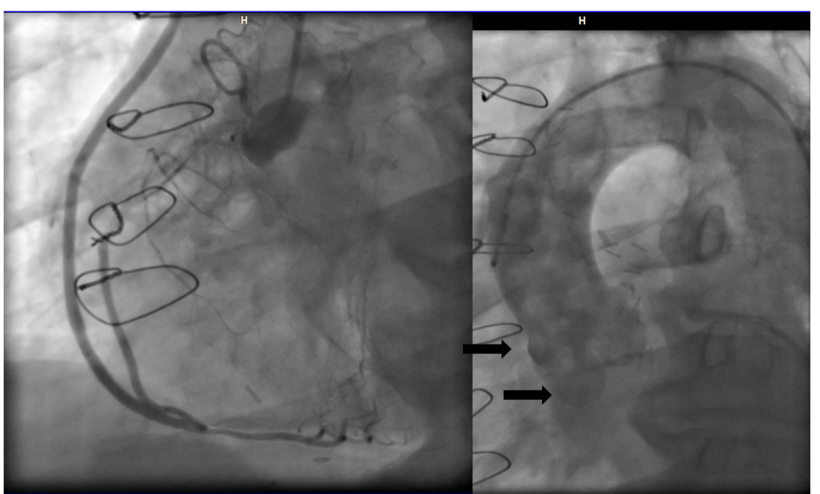

Figure 1. Left Anterior Oblique Projection Showing Spontaneous Aortocoronary Dissection (ACD) After Right Coronary Contrast Injection (Affected Aortic Root Stained With Contrast).

Valsalva was stained with contrast agent, and the border of the stained area was extended to the sinotubular junction. A sapheneous graft anastomosed to the distal RCA was also patent. Medical follow-up was proposed by the heart team. The patient was transferred to the intensive care unit for medical follow-up. Computed tomography (CT) showed a localized $1.5 \mathrm{~cm}$ aortic root dissection originating from the RCA ostium and extending towards the sinotubular junction (Figure 2). After that, daily echocardiographic imaging was performed (Figure 3 ). The dissection did not enlarge, nor did it progress. The patient was discharged five days later. Weekly echocardiographic follow-up continued for the first month, and a three-month control follow-up was also uneventful.

\section{Discussion}

Acute ACD is a rare clinical entity which occurs in $0.02 \%$ of diagnostic catheterizations and $0.07 \%$ of percutaneous coronary interventions. ${ }^{1}$ In the majority of cases, there is an intimal tear in the osteal segment of the coronary artery which subsequently leads to bidirectional dissection. An antegrade dissection of the coronary artery may extend to even the distal segment of the RCA, whereas, a retrograde dissection of the ascending aorta may lead to urgent clinical picture including cardiovascular collapse. Risk factors for catheter-induced aortic dissection were defined as follow: left main coronary artery intubation, use of special catheters, aggressive handling of rigid guide wires, catheterization during acute myocardial infarction and profound cannulation of the catheter into the coronary ostia. ${ }^{1}$ Risk factors for contrast-induced ACD, however, are not well defined because of its extremely rare incidence. Diffuse atherosclerotic disease, a rigorous injection rate, and adherence of the catheter's tip to the intimal layer seem to be major predictors of ACD. The treatment strategy depends mainly on the symptomatology of the patients. Stenting of the coronary ostium to seal the primary entry tear, thereby allowing the aortic dissection to heal spontaneously, has been reported as the best treatment option. ${ }^{1}$ However, surgical repair may be indicated in the propagation of dissection and hemodynamic instability. ${ }^{4}$ Conservative treatment of uncomplicated retrograde

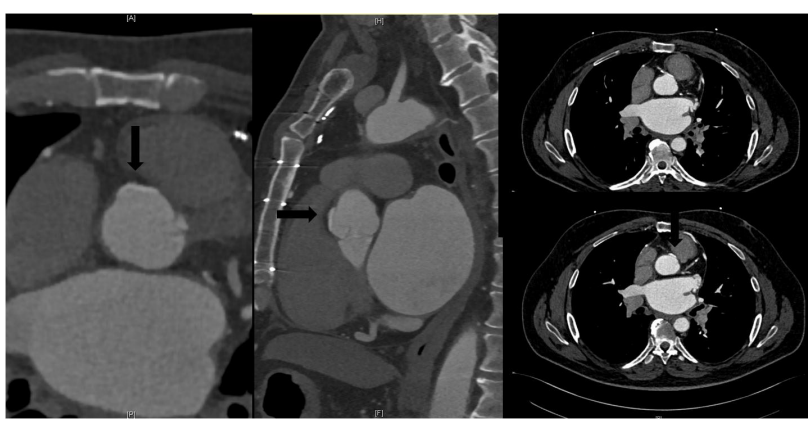

Figure 2. Computed Tomographic images Showing Localized Dissection of Aortic Root (Arrow indicates Dissected Area).

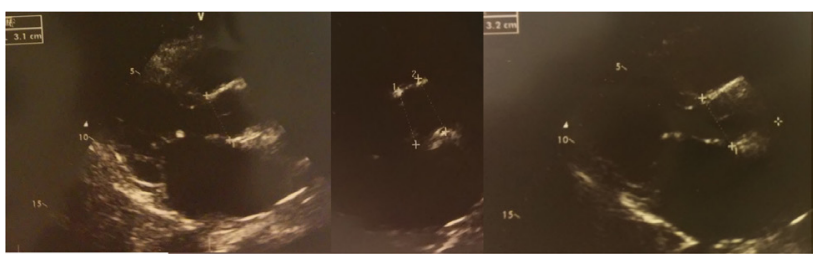

Figure 3. Echocardiographic image of Parasternal Short-Axis View Showing Aortic Root

dissection with blood pressure control, analgesics, and close observation has been recommended, especially in high-risk patients or those with previous cardiac surgery. ${ }^{2,5}$ Although a CT scan is the best modality for conservative follow-up, it brings additional contrast load and radiation exposure as well as an increase in costs. ${ }^{7}$ Conservative follow-up with echocardiogaphy as an appropriate imaging technique is suggested in such cases. It has the advantage of being a safer profile which does not necessiate additional radiocontrast agent and $\mathrm{x}$-rays. It is also cost affordable compared with CT scan and/or magnetic resonance imaging (MRI). However, echocardiographic examintion is self-dependent and requires experienced operators to avoid underdiagnosis. CT scan may give more accurate results in critical patients prior to deciding on surgical treatment. ${ }^{6,7}$

\section{Conclusion}

Iatrogenic $\mathrm{ACD}$ is a catastrophe in interventional cardiology. Conservative follow-up with imaging technique is proposed for small uncomplicated dissections. Computed tomography has been proposed as the gold standard for conservative follow-up; however, echocardiographic observation may show more satisfactory evidence than a CT scan in selected cases. Echocardiography also has the advantages of being of average cost, accessible, and safe. The authors consider conservative follow-up with echocardiogaphy to be the most appropriate imaging technique for use in small ACDs.

\section{Conflicts of Interest Disclosures}

The author reports no conflicts of interest regarding this work.

\section{Ethical Approval}

The work was approved by the Ethics Committee of the local hospital. 


\section{References}

1. Geomez-Moreno S, Sabate $M$, Jimenez-Quevedo $P$, et al. latrogenic dissection of the ascending aorta following heart catheterization: incidence, management, and outcome. Eurolntervention. 2006;2:197-202.

2. Shah P, Bajaj S, Shamoon F. Aortic dissection caused by percutaneous coronary intervention: 2 new cases reports and detailed analysis of 86 previous cases. Tex Heart Inst J. 2016;43:5260.

3. Huenges K, Dreyer J, Panholzer B, et al. Iatrogenic catheterinduced acute aortic dissection type A after coronary angiography - A retrospective consecutive case series. Thorac Cardiovasc Surg. 2016. doi:10.1055/s-0036-1594290.

4. Cebi N, Tanriverdi S, Karabulut A.latrogenic aortic dissection during right coronary artery stenting. Heart Surg Forum.
2008;11(3):E172-4.

5. Timek TA, Hooker R, Patzelt L, Bernath G. Conservative management and resolution of iatrogenic type $A$ aortic dissection in a patient with previous cardiac surgery. J Thorac Cardiovasc Surg. 2012;144:e18-e21. doi:10.1016/j. jtcvs.2012.05.007.

6. Geske JB, Pochettino A, Maltais S. Spontenous resolution of iatrogenic ascending aorta dissection: now you see it, now you do not. Eur Heart J. 2016;37(25):1984. doi:10.1093/eurheartj/ ehw175.

7. Neo WT, Pua U, Lee YS. Catheter-induced aorto-coronary artery dissection:utility and appearance on CT coronary angiogram. Int J Cardiol. 2010;144(2):334-337. doi:10.1016/j. ijcard.2009.03.038. 